\title{
Microstructure and mechanical properties of free and restrained dried paper: a comprehensive investigation
}

\author{
Georg Urstöger · Artem Kulachenko • Robert Schennach • Ulrich Hirn
}

Received: 28 February 2020/ Accepted: 23 July 2020/Published online: 28 August 2020

(C) The Author(s) 2020

\begin{abstract}
The influence of strain during drying is known to have a large effect on its mechanical properties like tensile strength, Young's modulus and hygroexpansion. In this study we investigate free and restrained dried paper and investigate the relation between the paper microstructure and its mechanical properties. The first part of the work investigates the development of the mechanical properties as well as paper internal stresses and strains (elastic-, inelasticand hygrostrain) at different moisture contents during the drying process. Emphasis is put on the changes of hygrostrains and the paper hygroexpansion coefficient
\end{abstract}

Electronic supplementary material The online version of this article (https://doi.org/10.1007/s10570-020-03367-4) contains supplementary material, which is available to authorized users.

G. Urstöger · U. Hirn ( $\square)$

Institute of Bioproducts and Paper Technology, TU Graz, Inffeldgasse 23, 8010 Graz, Austria

e-mail: ulrich.hirn@tugraz.at

G. Urstöger · A. Kulachenko · R. Schennach · U. Hirn CD Laboratory for Fiber Swelling and Paper Performance, Inffeldgasse 23, 8010 Graz, Austria

\section{A. Kulachenko}

Department of Engineering Mechanics, Royal Institute of Technology, 10044 Stockholm, Sweden

R. Schennach

Institute of Solid State Physics, TU Graz, Petersgasse

16/2, 8010 Graz, Austria during drying. One main finding was that in constrained drying the drying stresses are considerably below the yield stress and, as a consequence, the deformations are mainly inelastic (creep) and only marginally elastic. In the second part we are analyzing the microstructure of free and restrained dried sheets by X-ray microtomography $(\mu-\mathrm{CT})$. From the $\mu$-CT analysis relevant network parameters such as number of contact points, fiber bond area, free fiber length and fiber curl were extracted. Also a novel method to investigate the interface angle of the fibers in the vicinity of the bond sites was established. The results showed that the major difference from the drying procedure manifests itself in the out-of-plane curl of the fibers. Statistically number of contact points, bond area and in-plane curl were not different whereas inplane curl and free fiber length were different. The interface angle appears to be slightly affected by the drying procedure but is overall very low.

Keywords Drying - Mechanics - Microstructure Paper $\cdot$ Microtomography $\cdot$ Interface angle

\section{Introduction}

Depending on whether the paper is dried freely or under constraint, the resulting product shows very dissimilar mechanical properties, even at the same 
density. In particular, the elastic properties, strength, strain-to-failure and hygroexpansion coefficients depend on the paper strain during drying. Mäkelä (2009) The effects on the paper that are introduced by the removal of water are attributed to the changes in the fiber dimensions and its transfer across the network through the fiber bonds. The most recent review of the various factors affecting the hygroexpansivity of paper was presented by Lindner (2018). The largest contribution to the hygroexpansivity has long been attributed to changes in the individual fiber dimensions in the transverse direction during drying. One of the pioneering works with direct observation of the transverse hygroexpansivity of softwood pulp fibers with the help of micro-radiography was presented by Tydeman et al. (1966). Despite the essentially twodimensional observation allowing the visual detection of the fiber width change only, the authors also used the optical density of the image on the radiograph to correlate it with the fiber thickness change. By doing this, they could observe both the width and thickness change. The reported values for the fiber width shrinkage averaged around $30 \%$ for the beaten pulp and $20 \%$ for unbeaten pulp from fully soaked to dry state and were widely used since then. It was also observed that the thickness of the fibers contracted even more during drying, which was explained by the collapse of the fiber structure. The changes in the fiber wall thickness for fibers with open lumen could not be detected at that time. The fiber wall thickness was later reported to be an important parameter with thinner wall thickness resulting in larger hygroexpansion. Pulkkinen et al. (2009), Uesaka and Moss (1997).

Elastic properties, strength and strain-to-failure as functions of drying constraints

The drying process changes the microstructure of the fiber networks and the constraints applied during drying affect both the load history experienced by the individual fibers and the bonds between them. The discussion around the effect of the drying centers on two issues: the fiber activation and the fiber stiffening. In the fiber activation, it is postulated that during constrained drying, the fiber segments are straightened and the amount of the material participating in loadcarrying increases. Nanko and Wu (1995) compared the effect of the constrained and free drying at the micro level by observing the free and bonded segments with SEM. The authors reported that in constrained drying, the free (unbonded) fiber segments experienced extension, which may be associated with straightening leading to "activation". Activation refers to the idea that straightened, pre-strained fiber segments make a greater contribution to the load carrying than the unstraightened and slacker segments. The higher E-modulus of the constrained drying can be explained by this network activation. In the unconstrained drying, the free fiber segments became shorter. At the same time, the bonded segments are shortened in both types of drying conditions. The fiber shortening is associated with compressive stresses, which, it is believed, can induce microcompression in the fibers. Haslach Jr (1996) The observations were limited, however, to the surface fibers, which are having a lower number of bonds per length. For the fiber stiffening hypothesis, Jentzen (1964) showed that the tensile stress applied to individual fibers during drying increases both, their stiffness and their strength. The fibers dried under tension had a lower microfibril orientation angle and were thus stiffer.

By varying the tension during drying, Kouko et al. (2014) could demonstrate that the elastic modulus increases with increased draw applied during drying. In fact, the elastic modulus was reported to be a linear function of the shrinkage for both uniaxial and biaxial drying. Wahlström et al. (2000), Mäkelä (2009) The applied draw during drying also decreases the strain to failure. When scaled with the elastic modulus, all the stress-strain curves for the material dried under varied tensions aligned along the same curve merely have different endpoints. The authors showed that the sheet shrinkage occurring during drying can be recovered through extended strain-to-failure. At the same time, the biaxial experiments showed that the tensile stiffness in either of the two directions of applied load is independent of the strain in the perpendicular direction. This observation collaborated with both the "network activation" and "fiber stiffening" mechanisms. At the same time, the out-of-plane properties were affected by the drying as was shown by Wahlström et al. (2000). The freely dried sheet was reported to have higher $\mathrm{ZD}$ directional (normal to paper surface) strength. This was attributed to the decreased bonded area which was evaluated through the light-scattering coefficient. Interestingly, the drying time did not play a significant role for the in-plane tensile properties and this even though the stress 
variation during drying has a large impact on the tensile properties, which still show linear correlation with the final shrinkage regardless of the drying history. Mäkelä et al. (2009).

Hygroexpansion coefficient as a function of drying constraints

Hygroexpansion is one of the properties of paper, which affects the performance of paper products in printing and packaging applications. Kulachenko (2011) The dimensional stability is an outstanding issue in the emerging applications such as printed electronics, where one of the very few solutions available to prevent it is the use of hydrophobic coatings. Gozutok et al. (2019) Traditionally, the hygroexpansion coefficient is defined as the slope of the hygrostrain versus moisture content, despite the embedded non-linearity in the definition of the moisture content given as a percentage of the water in the papers total weight.

Paper has larger hygroexpansion coefficients in the cross-direction (CD) than in the machine-direction (MD) and the difference increases with increased anisotropy. Intuitively, it is easy to interpret this observation through the fact that the fibers mainly shrink in the transverse direction. The hypothesis that the transverse shrinkage of the fibers predefines the hygroexpansivity of the fiber network becomes very appealing in the light of this fact. With the developed theoretical framework, based on the composite theory, Uesaka (1994) showed, that a large fraction of the hygroexpansion should come from the longitudinal deformation of the fibers. This was later confirmed by the 3D simulation of the fiber network by Motamedian and Kulachenko (2019) in which the expansion of the fiber can be selectively disabled. The significance of the longitudinal deformation of the fibers is indirectly confirmed by the fact that the hygroexpansion coefficient of fiber networks is nowhere near the transverse hygroexpansion of the fibers even in case of dense sheets. Further indirect evidence supporting the contribution made by the longitudinal hygroexpansion of the fibers is that the MD expansion/shrinkage takes place faster than $\mathrm{CD}$ expansion, which is probably connected with the delay needed for moisture to reach/ leave the bonds. Larsson and Wågberg (2008), Lavrykov et al. (2004), Niskanen et al. (1997).
Paper dried freely has a significantly larger hygroexpansion coefficient than paper dried under constraints. The higher hygroexpansion coefficient for the freely dried sheets has been explained by two factors: increased bonding and increased stress transfer. The latter is believed to be associated with the changed configuration of the bonded area. Using a micromechanical approach Uesaka and Qi (1994) combined with the earlier developed theory by Uesaka (1994), showed that if the fibers develop out-of-plane orientation in the bonded region, this can enhance the stress transfer and, therefore, results in larger hygroexpansivity of the entire network. By contrast, fibers stretched during drying will have a greater probability of forming connections without a significant out-ofplane orientation. Another explanation of the higher hygroexpansion coefficient and yet lower elastic modulus of freely dried paper was offered by Salmén et al. (1987). They attributed the difference to the orientation of the cellulose and hemicelluloses which, during free drying release the dried-in stresses and rearrange their structure, making it more reactive to moisture changes.

In this work, we examine the changes in the microstructure and the mechanical properties paper sheets undergo during different drying regimes. Since the mechanical response of paper is essentially defined by the fibers, fiber bonds, and the sheet structure, we will focus on the microscale incorporating these three essential components. The majority of the direct observations of the changes the fibers and the fiber bonds undergo during drying are limited to the surface (Nanko and Wu 1995; Tydeman et al. 1966) or to single fibers Joffre et al. (2016). However, the fibers located close to the surface are not representative since they have a significantly lower number of contacts compared to the fibers located inside the sheet and are few compared to the bulk fibers. Therefore, in this paper, we make a series of controlled experiments, in which we collect the insights about the processes occurring in a paper sheet on microstructure and connect them to the mechanical changes the paper undergoes during drying.

In the first part, we will present the continuous development of the mechanical properties during constrained and free drying. We are analyzing mechanical properties (tensile strength- and stiffness, hygroexpansion coefficient) as well as paper internal stresses and strains (elastic-, inelastic- and 
hygrostrain) at different moisture contents. In the second part, we will examine the obtained network structures through X-ray microtomography and collect the statistics about the dimension of the fibers, the number of fiber bonds and their geometrical configurations. Through these observations, we will reflect on the changes induced by different drying and how they can be connected to the macroscale differences observed in the mechanical properties.

The assembled experimental material is shared as open data and can be served as a verification or calibration material for the micromechanical models Bosco et al. (2015), Motamedian and Kulachenko (2019), Salminen et al. (2002), Sellén and Isaksson (2014) as well as for training the machine learning algorithms to identify the fibers in micro-CT datasets, which are among the emerging techniques for the analysis of paper products.

\section{Material and methods}

\section{Sample preparation}

Paper lab sheets were produced in a Rapid-Köthen sheet former using a once dried, unbeaten, unbleached kraft pulp (Kappa 42) from an industrial producer. The chemical composition of the pulp was retrieved from one of the production batches similar to the one used in the study. The mass fractions of the pulp were found as: Cellulose 77\%, Hemicelluloses 16.6\% (Mannose $6.8 \%$, Xylose $8.5 \%$, Galaktose $0.5 \%$, others $0.8 \%$ ). Carbohydrate composition of the pulp was determined by acid hydrolysis of the pulp and subsequent HPLC. Lignin content was $6.3 \%$, converted to a mass fraction from Kappa number measurement. Extractives content was $0.1 \%$, measured by acetone extraction. The pulp was given at least $24 \mathrm{~h}$ to swell in deionized water before it was disintegrated to ensure a complete disentangling of the fiber clusters. After the sheetforming the lab sheets were wet-pressed. After wetpressing the lab sheets were kept between the covering papers and inside a plastic bag to prevent further drying. We produced lab sheets with a grammage of approx. $60 \mathrm{~g}$ applying a pressure of 5 bar for $30 \mathrm{~s}$. The measured properties of the sheets can be seen in Table 1.

Rectangular samples were prepared from the lab sheet by cutting around a template with a scalpel. The samples size was $20 \times 80 \mathrm{~mm}$ but with a strip of $15 \mathrm{~mm}$ on each side used for clamping with the result that the total length of the measured sample amounted to $50 \mathrm{~mm}$. During handling the samples were repeatedly sprayed with water to ensure that they did not exhibit any drying before being mounted on the tensile tester.

\section{Experimental setup}

Tensile measurements were performed on a tensile testing machine from Zwick/Roell (Type: BT1FB010TN.D30). The testing was carried out at $23^{\circ} \mathrm{C}$ and $50 \% \mathrm{RH}$ using a $500 \mathrm{~N}$ load cell. The samples were mounted at approximately $55 \%$ moisture content (MC) and air dried to $(8 \pm 1) \% \mathrm{MC}$. The moisture of the samples was established by measuring reference samples on a scale (OHaus, Type: PA213C) next to the tensile measurement.

The measurement routines were labeled as free and constrained to distinguish between free drying and drying under constraint, respectively. In the free drying case, the machine kept the force constant by adjusting the displacement while in the restrained drying case the machine kept the displacement constant and measured the force. It should be mentioned that the lateral restraints were not applied in either case. In the freely dried samples, a tension of $5 \mathrm{~N} / \mathrm{m}$ was maintained during drying to straighten the sample (which is approx. $3 \%$ of the final force attained during constrained drying). During constrained drying, the samples were adjusted to a pre-strain of $(0.08 \pm 0.05) \%$ to avoid slackness of the measured sheets. For both routines 8 samples were investigated.

E-modulus measurements were carried out for the free and constrained drying case. All the tests were performed according to the ISO 1924-A3 norm for tensile testing. In our case we used $50 \mathrm{~mm}$ long samples as it was closer to our free and constrained dried samples. For the free drying the samples were put on a scale and left to dry under standard conditions $\left(23^{\circ} \mathrm{C}, 50 \% \mathrm{RH}\right)$. When the desired moisture content (MC) was reached the sample was mounted and the tensile test was performed. For the constrained drying test, the samples were dried in the regions of the clamping before mounting in the tensile tester. Subsequently the samples were mounted and air dried under constraint until a desired MC was reached and the tensile test was performed. Immediately after the 
Table 1 Relevant parameters of the studied papers

\begin{tabular}{lllll}
\hline Sample & Grammage $/ \mathrm{g} / \mathrm{m}^{2}$ & Thickness $/ \mu \mathrm{m}$ & Apparent density $/ \mathrm{kg} / \mathrm{m}^{3}$ & Intrinsic density $/ \mathrm{kg} / \mathrm{m}^{3}$ \\
\hline Constrained & $55 \pm 3$ & $205 \pm 9$ & $269 \pm 5$ & $319 \pm 5$ \\
Free & $55 \pm 3$ & $235 \pm 11$ & $233 \pm 4$ & $307 \pm 7$ \\
E-modulus & $56 \pm 1$ & $231 \pm 3$ & $240 \pm 2$ & $320 \pm 2$ \\
\hline
\end{tabular}

The errors are indication of $95 \%$ confidence limits

test, the sample was unmounted and the dry clamping regions of the sample were cut off so only the measured area was weighted, and the quantitative MC could be evaluated.

A series of lab sheets was produced and caliper measured for investigating the intrinsic density of the papers according to standard EN ISO 534. After the measurement of the wet papers one group was dried under constraint while the other was dried freely. The intrinsic density of the sheets was evaluated by measuring the slope of a plot caliper over grammage of the sheets (Fig. 10). Intrinsic density is not affected by artifacts of surface topography such as apparent density as shown by Keller et al. and therefore reflects the true density of the fiber network (Sung et al. 2008).

The hygroexpansion coefficient was determined by two different methods. First (for the freely dried case) it was derived from the hygrostrain by simply taking the first derivative with respect to moisture content. Secondly it was determined by moisture cycling using a dynamic mechanical analyzer (DMA 850) from TA Instruments. The samples were equilibrated at $23^{\circ} \mathrm{C}$ and $33 \% \mathrm{RH}$ for $30 \mathrm{~min}$ before being exposed to 3 moisture cycles. The cycles consisted of going to $80 \%$ RH for $75 \mathrm{~min}$ followed by $33 \% \mathrm{RH}$ for $75 \mathrm{~min}$. The moisture change rate was $2 \% \mathrm{RH} / \mathrm{min}$. To match the relative humidity to the moisture content an isotherm was recorded which can be found in ESI. The hygroexpansion coefficient $\beta_{\text {hygro }}[-]$ was calculated via

$\beta_{\text {hygro }}=\frac{\epsilon^{\text {high }}-\epsilon^{\text {low }}}{M C_{\text {high }}-M C_{\text {low }}}$

where $\epsilon^{\text {high }}[\%]$ and $\epsilon^{\text {low }}[\%]$ refers to the hygrostrain after $75 \mathrm{~min}$ at $80 \% \mathrm{RH}$ and $33 \% \mathrm{RH}$, respectively. $M C_{\text {high }}$ [\%] and $M C_{\text {low }}$ [\%] refer to the moisture content after $75 \mathrm{~min}$ at $80 \% \mathrm{RH}$ and $33 \% \mathrm{RH}$, respectively. Sample population sizes for Const MD, Const $\mathrm{CD}$ and Free were 5,4 and 3, respectively.
Moisture development during drying

Figure 1 shows schematically how the recorded mechanical parameters such as force (Fig. 1a) and moisture (Fig. 1b) developed as a function of time. It shows that the drying stresses started to develop at around $50 \% \mathrm{MC}$ as the free water disappears and the fibers start shrinking. In Fig. 1c one can see how the plots from (a) and (b) were combined to a force versus moisture diagram. Drying strain versus moisture was evaluated accordingly.

Fiber characteristics and network analysis

After performing the mechanical measurements two type of samples, biaxially constrained and freely dried were taken and investigated by X-ray microtomography using a Zeiss Xradia XRM 520 high resolution lab $\mathrm{X}$-ray tomograph. The region of interest was a size of approx. $1 \mathrm{~mm}^{2}$ and the voxel size was $700 \mathrm{~nm}^{3}$. Two fully constrained samples and one fully free sample were investigated.

The obtained data was analyzed by a Matlab script developed originally by Wernersson et al. (2014) and is schematically shown in Fig. 2. A detailed description on how all parameters are extracted from the data can be found in Borodulina et al. (2016). The extracted parameters include data such as the length of the individual fibers, number of contact points (NCP), free fiber length (FFL) and curl. Also, the overlap area between the fibers was evaluated yielding an estimate of the bond area. From the positions of the bonds along the fiber the free fiber length was calculated. Additionally, the positions of the fibers were detected so that the network could be remodeled in exactly the same way as it was during the mechanical measurement (Fig. 2d). 

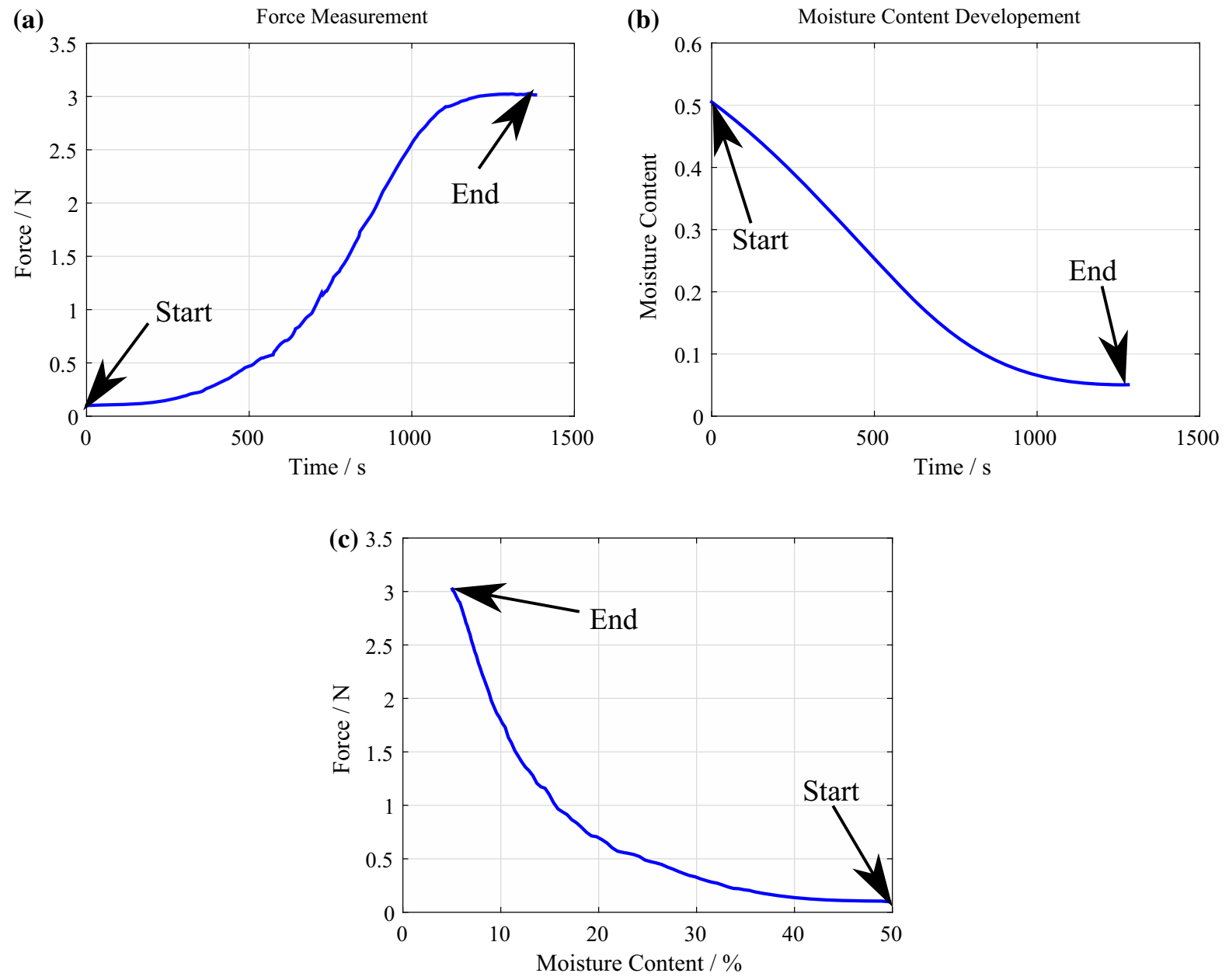

Fig. 1 Description how data of (a) and (b) were combined to (c) drying stress over moisture content

Interface angle

The fiber data was analyzed to evaluate the interface angle around the bond sites of the network. This is shown schematically in Fig. 3. The bending of the fibers at a bond site can in principle fall into one of three categories. In (a) both ends of fiber 1 bend towards fiber $2\left(\beta_{1,2}>0\right)$. In (b) both ends of fiber 1 bend away from fiber $2\left(\beta_{1,2}<0\right)$. Case (c) is a mixed case in which one end points towards- and the other end away (one $\beta$ positive and the other $\beta$ negative) from the bond. We constructed a triangle for every bond for the analysis as in Fig. 3a. The directionality angle $\alpha$ determines whether a side is pointing towards $\left(\alpha<90^{\circ}\right)$ or away $\left(\alpha>90^{\circ}\right)$ from the fiber bond. In our analysis an interface angle $\beta$ was defined as positive if $\alpha<90^{\circ}$ and negative if $\alpha>90^{\circ}$. The interface angle $\beta$ was calculated using the start-, center- and endpoint of each bond.

\section{Shared data}

All the data presented in this work has been uploaded and can be used by other authors for any purposes (given that this work is attributed appropriately). The data and its detailed description can be found in the electronic supplementary information in the form of a zip file labelled Data_repository. $7 z$. 
(a)

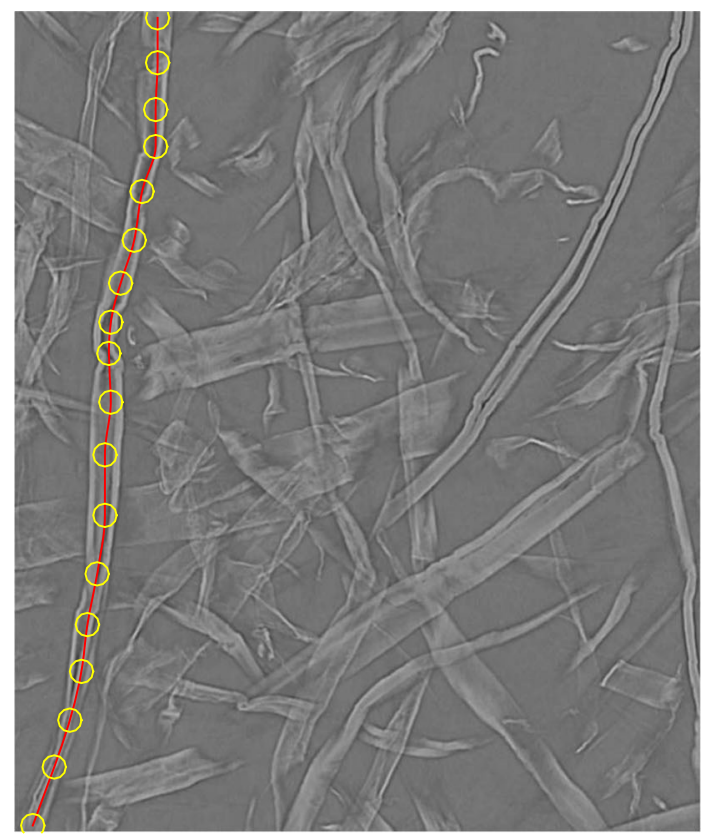

Fig. 2 Schematic diagram of how the $\mu$-CT data was extracted. In the tomography images individual fibers were detected in a xy dimensions and subsequently in $\mathbf{b} \mathrm{xz}$ dimension. The identified (b)

(c)
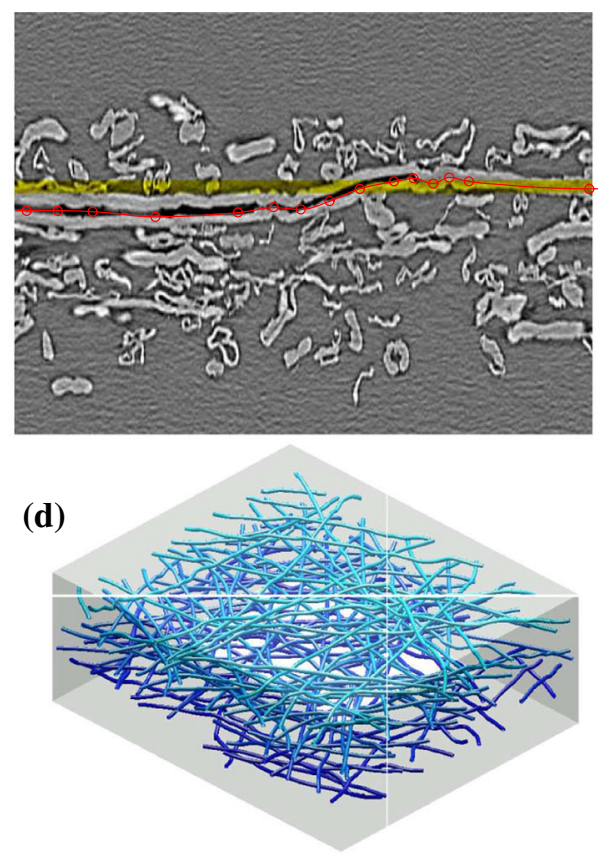

fibers were then followed along their centerline. The cross sections along the fibers were then used to determine fiber bonds and estimate the bonded area. d Shows a reconstructed network (a)

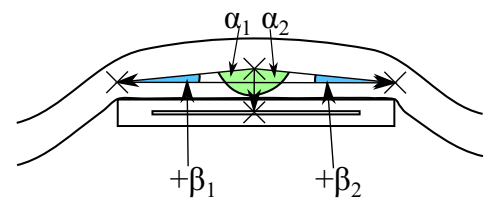

(b)

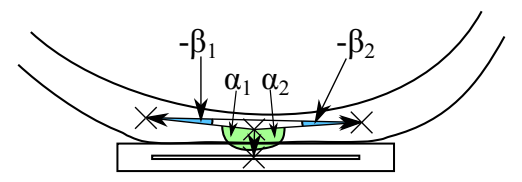

(c)

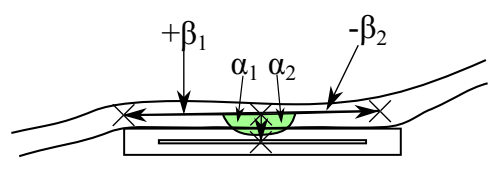

Fig. 3 Schematic diagram of the different possibilities for fiber bonds. a The top fiber bends on both bond edges towards the bond (Case a, $\beta_{1,2}>0$ ). b Top fiber bends on both bond edges away from the fiber bond (Case b, $\beta_{1,2}<0$ ). Or the intermediate

\section{Results and discussion}

The results section will be split into two parts. First the mechanical investigations and second the structural investigations.

\section{Part I: Mechanical investigations}

\section{Development of the E-modulus}

The first property that was measured was the E-modulus. The trend can be seen in Fig. 4. E-modulus was derived from the tensile tests accumulated in Fig. 5a case $\mathbf{c}$ in which one end of the top fiber bends towards- and one away from the bond (one $\beta$ positive and the other $\beta$ negative)

and $b$. Overall low values for the E-modulus are expected as the pulp is unbeaten and the paper has a low density (see Fig. 10 and Table 3). The development of the E-moduli can be described by an exponential fit with an additional offset (Eq. 2) to account for the wet web strength. Wahlström (2004) The fit parameters can be seen in Table 2 .

$$
y(M C)=a \cdot e^{-b \cdot M C}+c
$$

The results show that the constrained drying increased the elastic modulus by a factor of 3-4 compared to the free drying at the same level of anisotropy and with the 


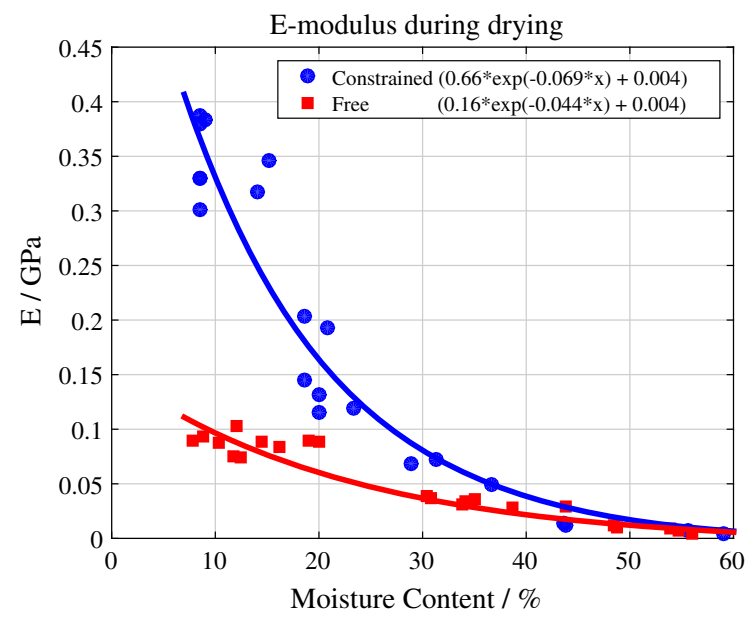

Fig. 4 Development of E-modulus during drying. The behavior is fitted according to Eq. 2

\section{Constrained}

(a)

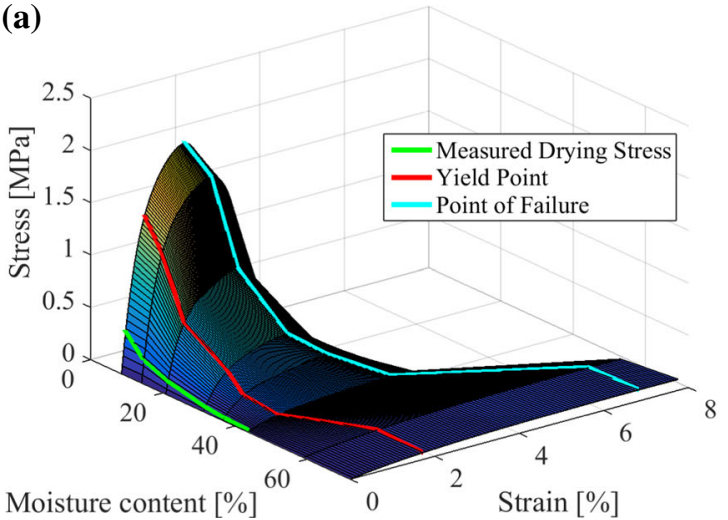

(c)

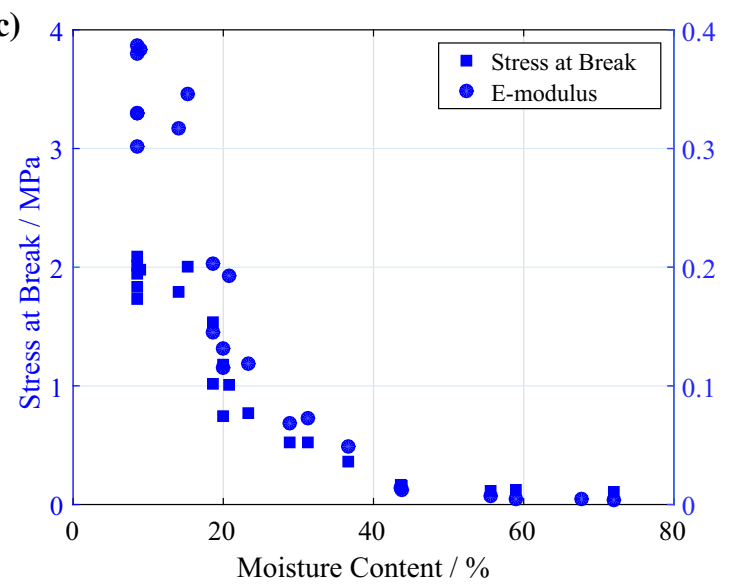

Fig. 5 Tensile tests of constrained (a) and free paper (b) at different MC levels and the corresponding stress at break, and E-modulus (c, d). In the constrained data (a) the hygro-induced
Table 2 Parameters for exponential fit of E-modulus

\begin{tabular}{lllll}
\hline Method & $\mathrm{a} / \mathrm{GPa}$ & $\mathrm{b} /-$ & $\mathrm{c} / \mathrm{GPa}$ & $R^{2}$ \\
\hline Const & $0.66(3)$ & $0.069(4)$ & $0.004(1)$ & 0.97 \\
Free & $0.159(6)$ & $0.044(3)$ & $0.004(1)$ & 0.94 \\
\hline
\end{tabular}

same fibers. This difference was to be expected, as discussed in "Elastic properties, strength and strainto-failure as functions of drying constraints" section of the introduction.

In Fig. 5a,b representative tensile tests for the evaluation of the E-modulus at different moisture contents are shown. In (a) the three lines indicate the measured drying stress for the constrained dried paper

\section{Free}
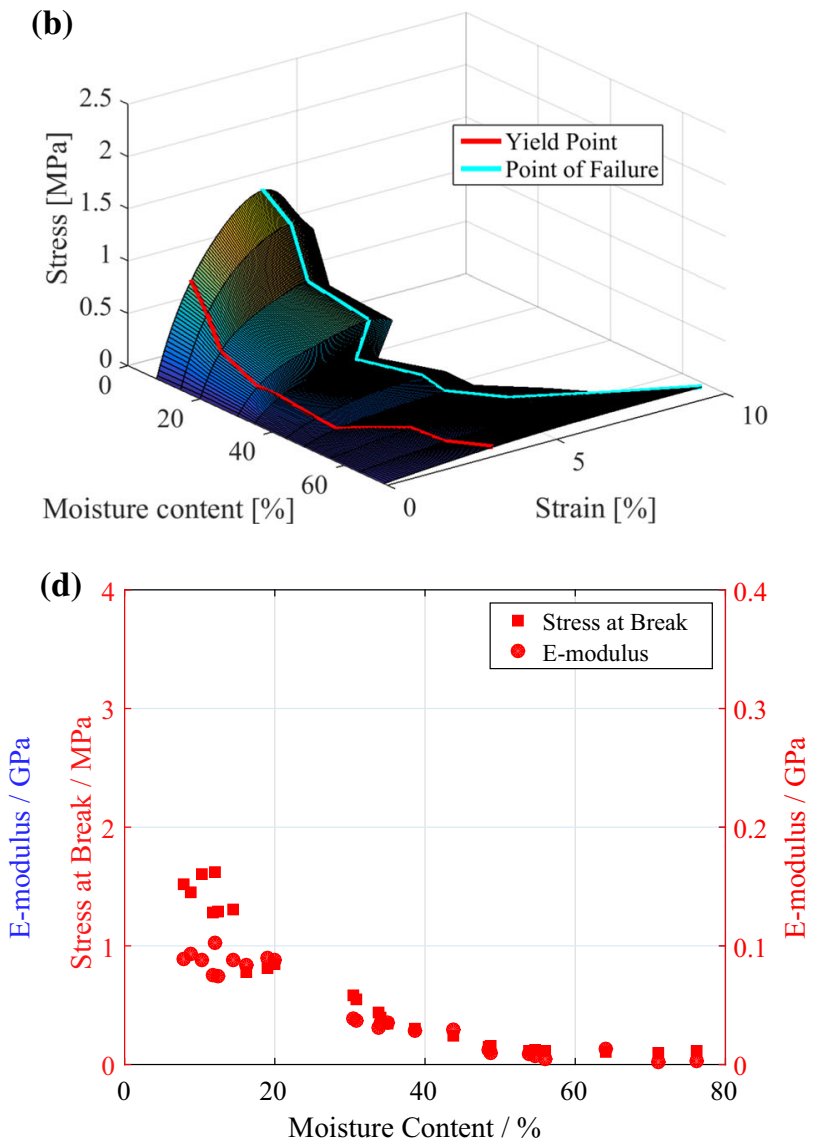

stress from Fig. 6a and the offset yield strength are indicated, showing that the drying stress for constrained drying is considerably below the yield stress 

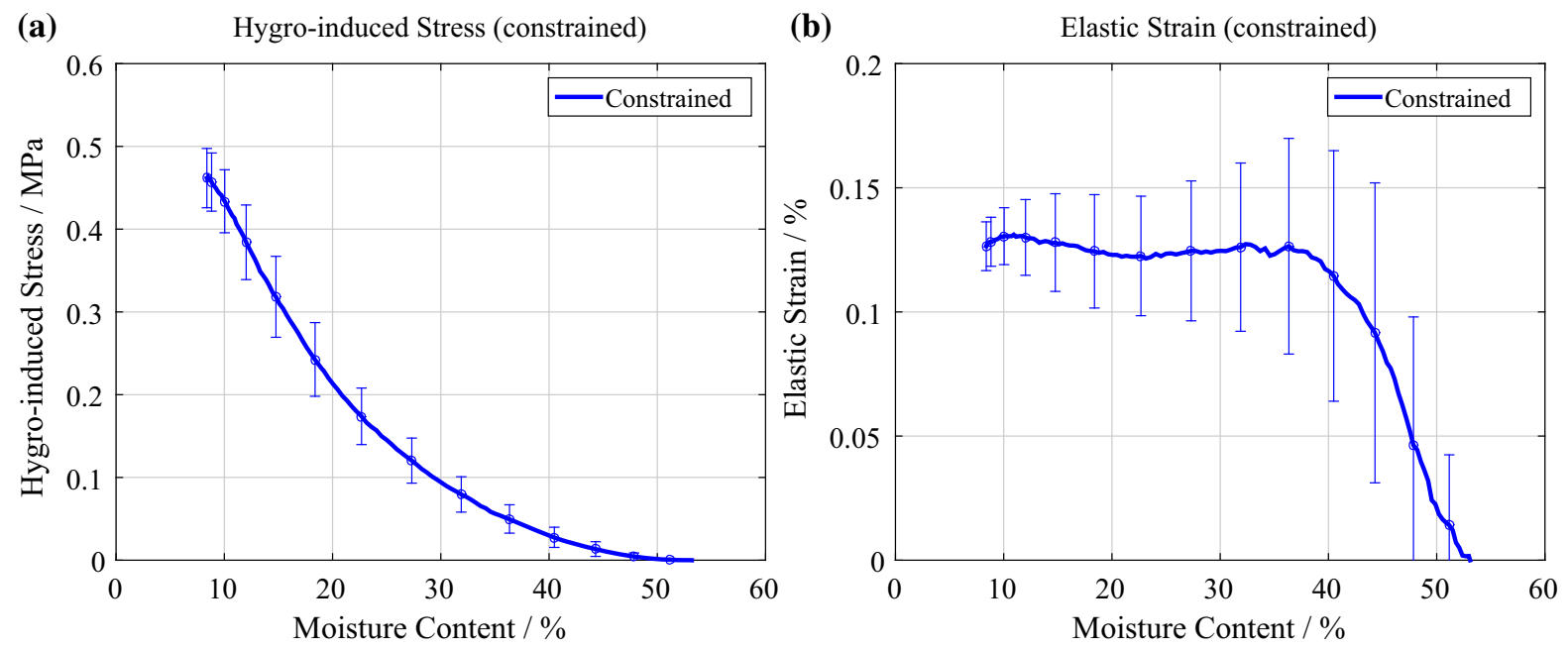

Fig. 6 Hygro-induced stress (a) and elastic strain (b) for constrained drying. In constrained drying it is only possible to measure the developing stresses. Hence the strain is calculated by Eq. 3. Error bars are $95 \%$ confidence limits

(green), the offset yield point (red) and the point of failure (turquoise). One can see clearly that the drying stresses of constrained drying (green) are considerably below the yield stresses (red) of the paper. In (b) only the offset yield point and the point of failure is indicated as the free drying occurs by definition at zero stress hence no drying stresses originate. Additionally to the E-modulus, stress at break was evaluated. The trends can be seen in Fig. 5c,d.

\section{Stress and strain development during drying}

This section will provide the detailed results and the discussion on the developing strains and stresses during free and constrained drying. Due to the experimental procedure it is only possible to either measure the developing strain (free drying) or measure the increasing stress (constrained drying). Stress in the case of free drying and strain in the case of constrained drying was calculated via Eq. 3. The resulting data can be seen in Fig. 6.

$\sigma=\epsilon_{\text {elastic }} \cdot E$

With $\sigma$ as the stress $\left[N / m^{2}\right]$, E the Young's modulus $\left[N / m^{2}\right]$ and $\epsilon_{\text {elastic }}[-]$ the elastic strain.

The following discussion will provide insights in the obtained data and how it can be interpreted. Generally, strain in paper can be described by the following equation

$$
\epsilon_{\text {tot }}=\epsilon_{\text {elastic }}+\epsilon_{\text {hygro }}+\epsilon_{\text {inelastic }}
$$

Where $\epsilon_{\text {tot }}[-]$ is the total (cumulative) strain, $\epsilon_{\text {elastic }}$ [-] is the elastic strain which is defined as deformations leading to external forces, $\epsilon_{\text {hygro }}[-]$ is the strain due to interaction with moisture and $\epsilon_{\text {inelastic }}[-]$ is the sum of all the other inelastic strains such as creep or plastic deformation.

In the freely dried case the strain (Fig. 8a) is measured directly in the experiment. Its development starts slightly above 50\% MC and ends at a strain of about $3.5 \%$. In the case of free drying the external forces are 0 which leads to a vanishing elastic strain $\epsilon_{\text {elastic }}$ and Eq. 4 hence reduces to

$\epsilon_{\text {tot }}=\epsilon_{\text {hygro }}+\epsilon_{\text {inelastic }}$

During free drying the other inelastic deformations unrelated to hygrostrain will also be very small. Therefore, the strain of the freely dried sample in Fig. 8a is mostly direct hygrostrain and only to a very small portion inelastic strain. With this argumentation we can further reduce Eq. 5 to

$$
\epsilon_{\text {tot }}=\epsilon_{\text {hygro }}
$$

Thus we are measuring pure hygrostrain in Fig. 8a.

In the case of the constrained samples the elastic strain $\left(\epsilon_{\text {elastic }}\right)$ is calculated from the measured stress in Fig. 6a and the E-modulus from Fig. 4 by Eq. 3.

The elastic strain can be seen in Fig. 6b. The most important result from this Figure is the very small 
magnitude of the elastic strain. For a comprehensible explanation of this result we again start from Eq. 4. As we are performing a constrained measurement the total strain $\left(\epsilon_{t o t}\right)$ is zero which results in

$0=\epsilon_{\text {elastic }}+\epsilon_{\text {hygro }}+\epsilon_{\text {inelastic }}$

Since hygrostrains and other inelastic strains do not yield stresses by definition, the calculated strain in Fig. $6 \mathrm{~b}$ is the pure elastic strain in the paper that develops due to a changing moisture content. Eq. 7 can thus be written as

$-\epsilon_{\text {elastic }}=\epsilon_{\text {hygro }}+\epsilon_{\text {inelastic }}$

As the constrained paper is subjected to the same change in moisture as the freely dried one the resulting hygrostrain must also be the same as in the free drying case. As depicted in Fig. 7 and Eq. 7 this means that for the elastic strain to become as small as seen in Fig. 6 the inelastic strain must become almost as big as the hygrostrain. From an observational point of view this is exactly what has been described to occur in a paper during constrained drying. The hindering of the hygrostrain leads to strong inelastic deformations which manifest in the prestraining of fiber segments that lead to an increased E-modulus of the paper. Additionally, we know from Fig. 5 that during the

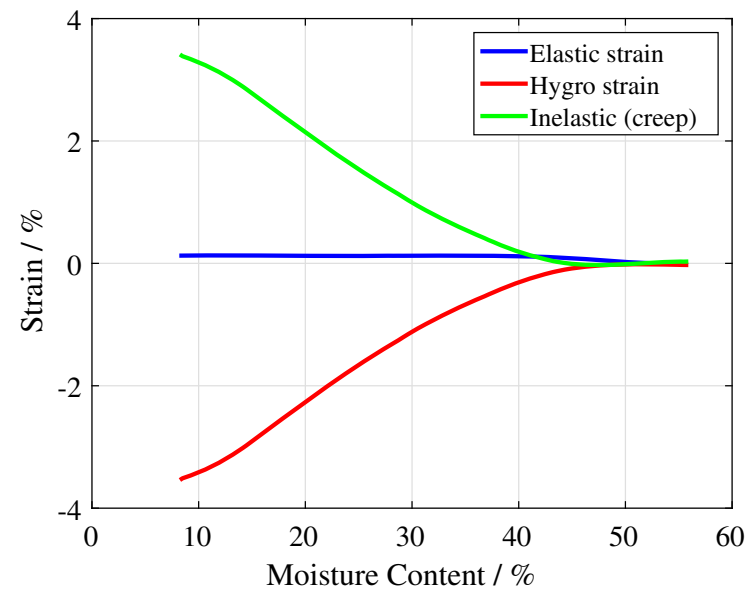

Fig. 7 Schematic for the large inelastic strains during constrained drying. The plot argues that the inelastic (creep) components in paper can become very large in constrained drying. The elastic strain, obtained by Eq. 3, is comparatively small. Starting with the proposition that the same amount of moisture influence occurs in both the free and the constrained case, we argue that the inelastic strains must reach a dimension that is nearly equal to that of the hygrostrain according to Eq. 8 active drying procedure drying stresses remain below the yield point. Thus, we conclude that in constrained drying of paper the strains that occur consist to a great extent of creep deformations and to a small extend of elastic deformations.

\section{Hygroexpansion}

Following on from the examination of the strains that develop in the paper during drying, the different hygroexpansion coefficients were also investigated. The hygroexpansion coefficient of the freely dried paper can be obtained directly by the derivative of the obtained hygrostrain over moisture content, Eq. 1. The results can be seen in Fig. 8. The curve is constant from (8-23)\% MC then decreases to approx. 52\% MC where it finally hits zero.

In Fig. 9 the hygroexpansion coefficient, determined from moisture cycling can be seen. The free and the unconstrained (CD) direction of the MD constrained paper follow the same trend. This is evidence that the constrained direction does not influence the unconstrained direction which was also observed by Wahlström et al. (2000).

\section{Part II: Structural Investigations}

\section{Density}

The density change of the paper during drying was tested and can be seen in Fig. 10. The reason for this measurement was the well-known interrelation between E-modulus and density.

The papers were dried from approx. $55 \% \mathrm{MC}$ to approx. $8 \% \mathrm{MC}$. As one can see in the grammagethickness plot the curves have nearly the same slope which means that the density of the paper does not change either during restrained or free drying. The inverse of the fitted slopes (i.e. the density) for the lines in Fig. 10 are listed in Table 3. However, a significant increase in the roughness of the paper, can be observed. The qualitative difference in roughness was also established by means of an analysis of the papers using light microscopy as seen in Fig. 11.

As can be seen in Table 3 the change in density from wet to free dried is small $(3 \%)$. There are two factors which can explain this observation. One is the fiber stiffness of the unbeaten pulp and the second is 

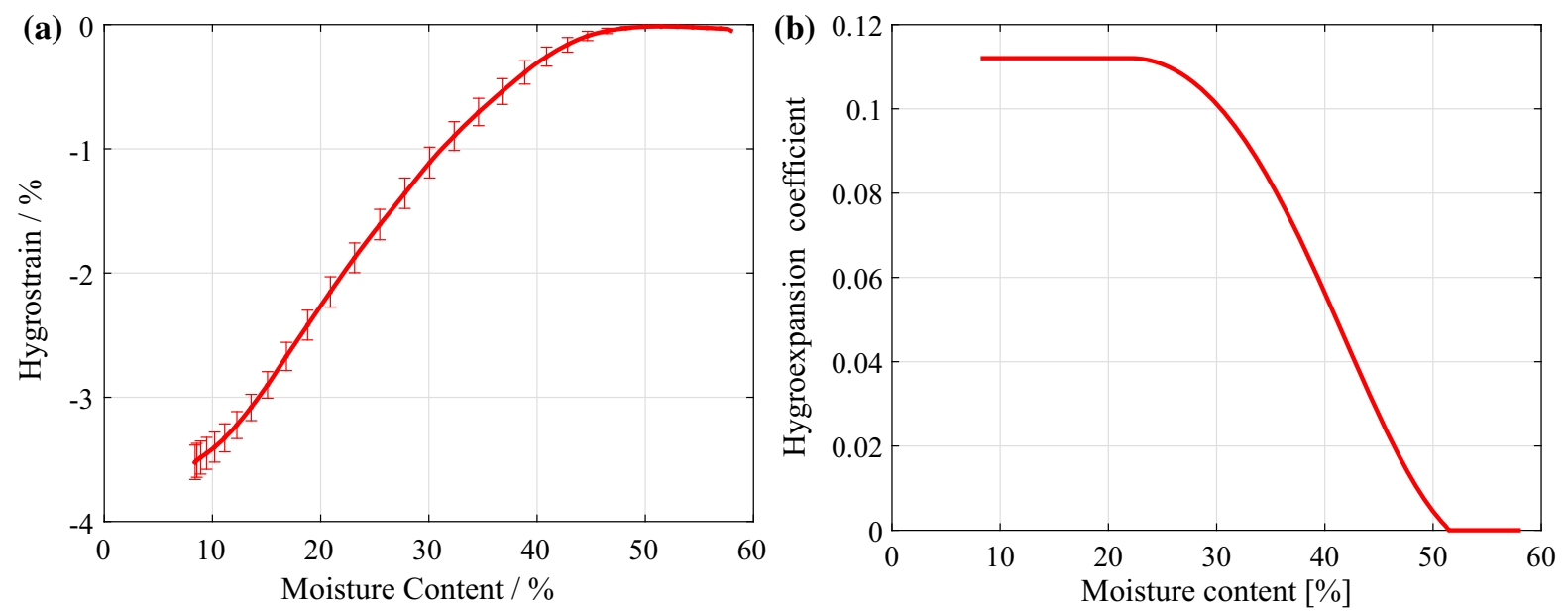

Fig. 8 Hygrostrain and hygroexpansion of the freely dried paper. a Shows the directly measured hygrostrain during drying. b Shows the hygroexpansion coefficient derived from (a). Error bars are $95 \%$ confidence limits

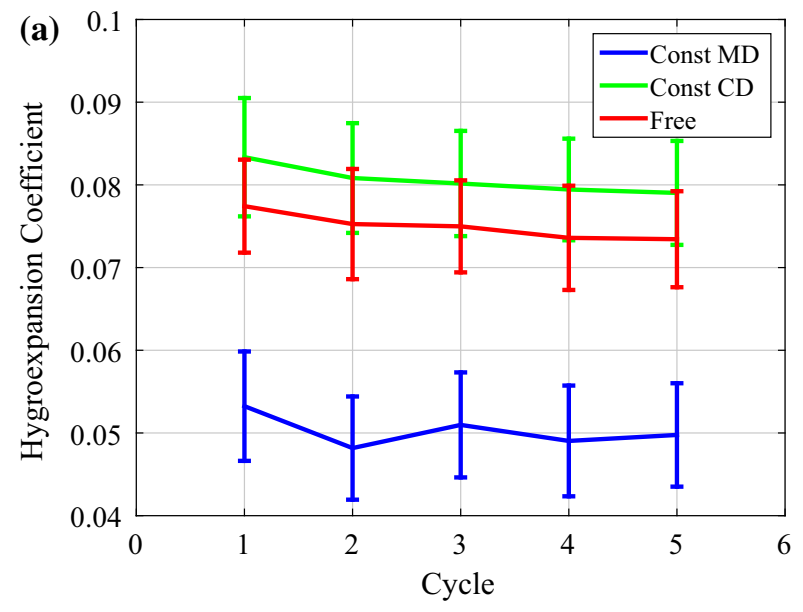

Fig. 9 Hygroexpansion coefficient (a) and hygroexpansion (b) of the constrained and freely dried papers from moisture cycling experiments. As expected the hygroexpansion is lower for the constrained dried paper. The freely dried paper and the

the drying procedure without lateral pressure. Two control experiments with beaten pulps and with a different drying procedure (standard Rapid Köthen drying) were performed to investigate the influence of fiber elasticity and the influence of the drying procedure, respectively. The data for these measurements can be found in the ESI. In all cases the lab sheets that were produced showed a decrease in thickness, hence, in our papers, the observed constant density is a combination of both factors, non-laterally constrained drying in air and an unbeaten pulp.

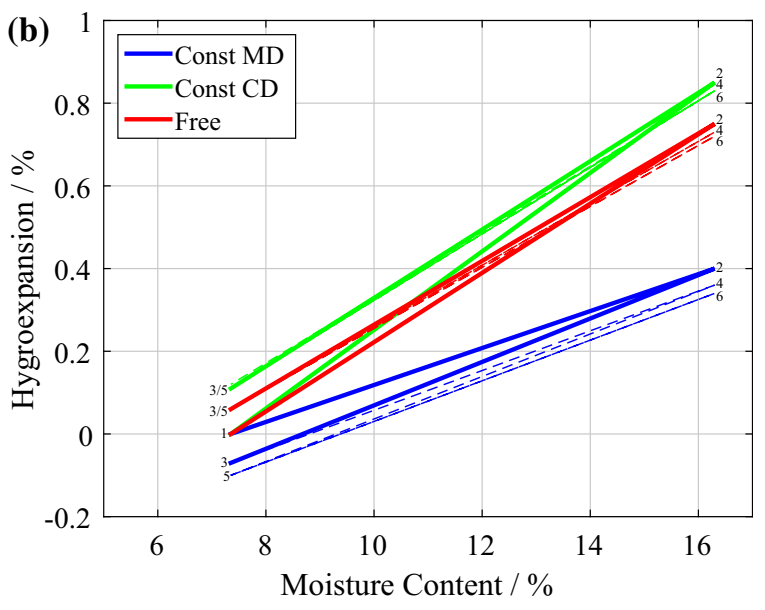

free direction of the constrained paper follow a similar trend. The results also compare well to the fit seen in Fig. 8. Error bars are $95 \%$ confidence limits

The change in roughness can be explained on the one hand by the measurement procedure and on the other hand by the drying procedures. The roughness of the wet paper is low because, using a standard caliper, the paper conforms more to the pressure due to increased compliance. In the free and the restrained case the difference comes most likely from the fibers. In the free case more fibers project out of the $\mathrm{z}$ plane and yield a higher roughness as a result. This is also in agreement with our results in the microstructural 


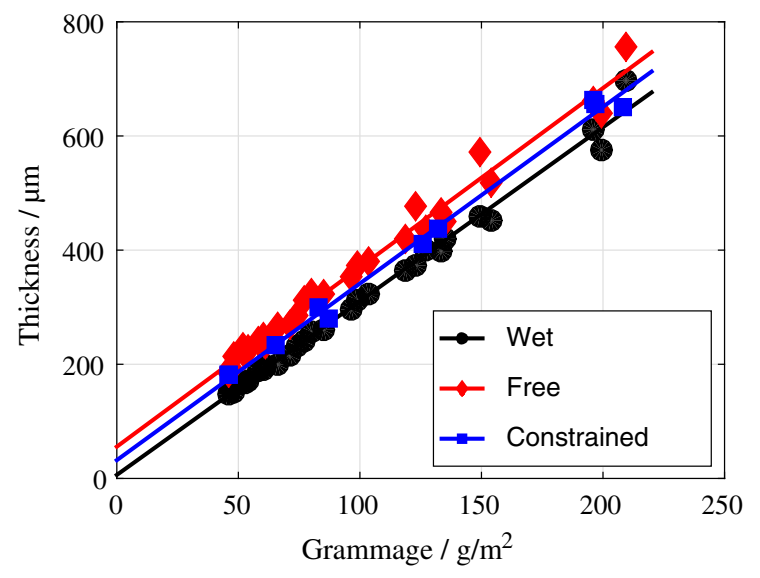

Fig. 10 Thickness over grammage of the two investigated drying strategies. As can be seen no significant density change (i.e. equivalent slopes) can be observed compared to the wet paper

Table 3 Density and roughness of the differently dried papers in Fig. 10

\begin{tabular}{llll}
\hline Method & Density $/ \mathrm{kg} / \mathrm{m}^{3}$ & Roughness $/ \mu \mathrm{m}$ & $R^{2}$ \\
\hline Wet & $328 \pm 12$ & $6 \pm 13$ & 0.99 \\
Free & $318 \pm 15$ & $56 \pm 18$ & 0.98 \\
Const & $324 \pm 20$ & $32 \pm 27$ & 0.99 \\
\hline
\end{tabular}

Errors are $95 \%$ confidence limits

investigation, since in this the free dried samples exhibit a higher out-of-plane curl.

\section{Microtomography analyses}

This section discusses the results obtained from the microtomography analysis of the constrained and freely dried papers. The methodology of how the data was extracted from the scans can be seen in Fig. 2. The number of contact points (NCP) and the Free Fiber Length per $\mathrm{mm}$ (FFL) were normalized by the density of the sheets and adjusted to a density of $300 \mathrm{~kg} / \mathrm{m}^{3}$ to make the results comparable to the other investigated papers of this work. The network statistics can be found in Table 4. Differences between distributions were analyzed using the Kolmogorov-Smirnoff test (ks-test).

The results for number of contact points (NCP) can be seen in Fig. 12a. It shows that the drying strategy does not have a significant effect on the NCP in the networks, see ks-test in Table 4. Although that the NCP in the papers are the same the free fiber length (Fig. 12c) is significantly $(p=0.002)$ lower in the constrained case as opposed to that for free drying. The bond area (Fig. 12b) also appears not to be affected by the drying procedure.

Furthermore the in- and out-of-plane curl of the fibers was investigated. As can be seen in Fig. $12 \mathrm{~d}$ and ks-test in Table 4 the in-plane (without $\mathrm{z}$ ) curl in both networks is equivalent. A significant $(p<0.001)$ difference arises when the $\mathrm{z}$-directional component of the curl is included. It becomes evident that a freely dried network exhibits a higher total curl than a constrained network and this difference stems from the difference in the out-of plane fiber curl.

Another highly discussed network parameter in relation to the effect of drying is the behavior of a fiber in the vicinity of a bond site. In schematic drawings of fiber-fiber bonds usually a configuration like Fig. 3a is shown, where fibers are curved towards the bond around the bonding region. For our analysis we also evaluated case $\mathrm{b}$, fibers curved away from the bonding region, and case c (one fiber end curved towards- and one fiber end curved away from the bonding region). Figure 13 gives the results, the labeling is according to Fig. 3. Interestingly enough there are almost as many fiber ends bending away from the bond $(-\beta)$ as there are bending towards the bond $(+\beta)$, the distribution is almost 50:50. Even more surprising is the preferred configuration of the fiber bending, more than $60 \%$ follow case $\mathrm{c}$ with one fiber end bending towards the bonding region and one fiber bending away from the bonding region. Cases $\mathrm{a}$ and $\mathrm{b}$ are nearly equally likely. In total we can conclude that the usual way fiber-fiber bonds are pictured, i.e. fibers bending towards the bonding region (case 1), is not supported by quantitative analysis. In fact there is no preferred directionality of the fiber curvature around fiber bonding regions, and the most likely case even is a mixed mode bending (case 3). Finally we find that there is almost no influence of the drying strategy on the interface angle $\beta$ between fiber and bond region.

Quantitative analysis of the fiber bending around bonding regions is provided by Fig. 14, again divided in cases a, b, c, according to Fig. 3. The statistics of these distributions can be found in Table 5. The distributions are similar for positive and negative interface angles. The bimodal form of the distributions is surprising, an unimodal distribution with $0^{\circ}$ as most 


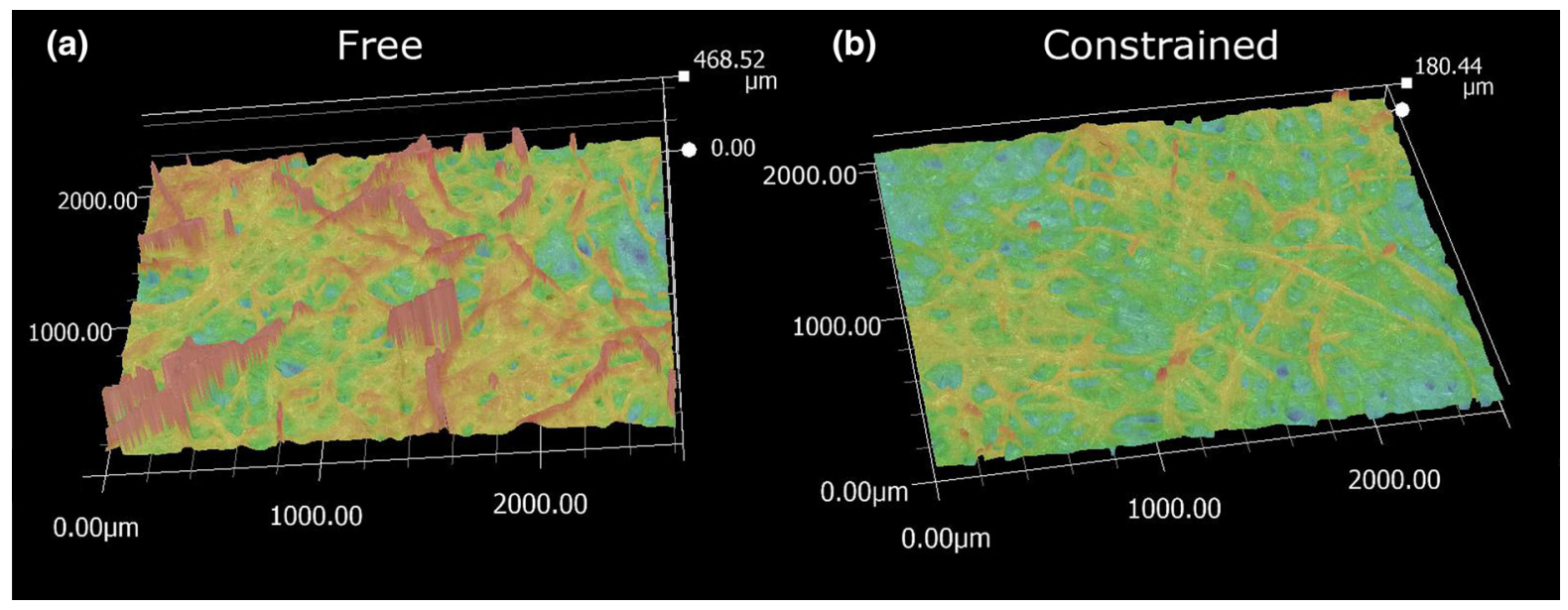

Fig. 11 Surface topography of the investigated paper dried under different conditions. The difference in roughness is clearly visible

Table 4 Statistics of the distributions seen in Fig. 12

\begin{tabular}{|c|c|c|c|c|c|}
\hline Free dataset & $\mathrm{NCP}$ & Bondarea/ $\mu \mathrm{m}^{2}$ & FFL per $\mathrm{mm} / \mathrm{mm}$ & Curl (without z) $/ \%$ & Curl (with z) $/ \%$ \\
\hline \multicolumn{6}{|l|}{ Network statistics } \\
\hline Mean & 13.4 & 590 & 0.54 & 3.3 & 6.0 \\
\hline Standard deviation & \pm 5.6 & \pm 450 & \pm 0.27 & \pm 5.2 & \pm 5.7 \\
\hline Error (95\% Conf.Int.) & \pm 0.87 & \pm 33 & \pm 0.03 & \pm 0.66 & \pm 0.72 \\
\hline \multicolumn{6}{|l|}{ Constrained dataset } \\
\hline Mean & 13.1 & 580 & 0.46 & 3.2 & 4.7 \\
\hline Standard deviation & \pm 6.9 & \pm 470 & \pm 0.27 & \pm 5.4 & \pm 5.8 \\
\hline Error (95\% Conf.Int.) & \pm 1.0 & \pm 43 & \pm 0.05 & \pm 0.99 & \pm 1.1 \\
\hline \multicolumn{6}{|c|}{ Kolmogorov-Smirnov test statistics } \\
\hline Test decicion & Equivalent & Equivalent & Different & Equivalent & Different \\
\hline$p$ value & 0.23 & 0.08 & 0.002 & 0.37 & $<0.001$ \\
\hline
\end{tabular}

The distributions were tested for difference between constrained and free drying using a Kolmogorov-Smirnoff test

frequent value would be more intuitive. As far as we checked this is not an artifact from from e.g. discretization, it seems to be present in the data. We also find that the interface angles in general are quite small, usually below $15^{\circ}$. The cases make different contributions to the distribution. The majority of the small interface angles come from the bonds of case $c$. The interface angles in case a (fiber curved towards bond) and $b$ (fiber curved away from bond) have similar values, however they are considerably larger than for case $\mathrm{c}$.

In Fig. 15 we are first analyzing if the fiber interface angle, i.e. the curvature angle around fiber bonds, is higher than the average curvature of the fibers. In other words we want to evaluate if the bonding region is affecting the local curvature of the fiber. For that we calculated the angle between different fiber segments disregarding the bond sites. The length of the fiber segments was chosen as the average length of a bond in the network. The data was fitted to a pdf using an unbiased kernel distribution (ks density function in MATLAB). We find that the Interface angles over the fiber fiber bonds (solid lines) have slightly higher values than the fiber segment angles between the bonds (dashed lines), indicating a slightly higher curvature of the fiber in the bonding regions. This would indicate that there is a small yet measurable bending deformation of the fibers due to a bond. 

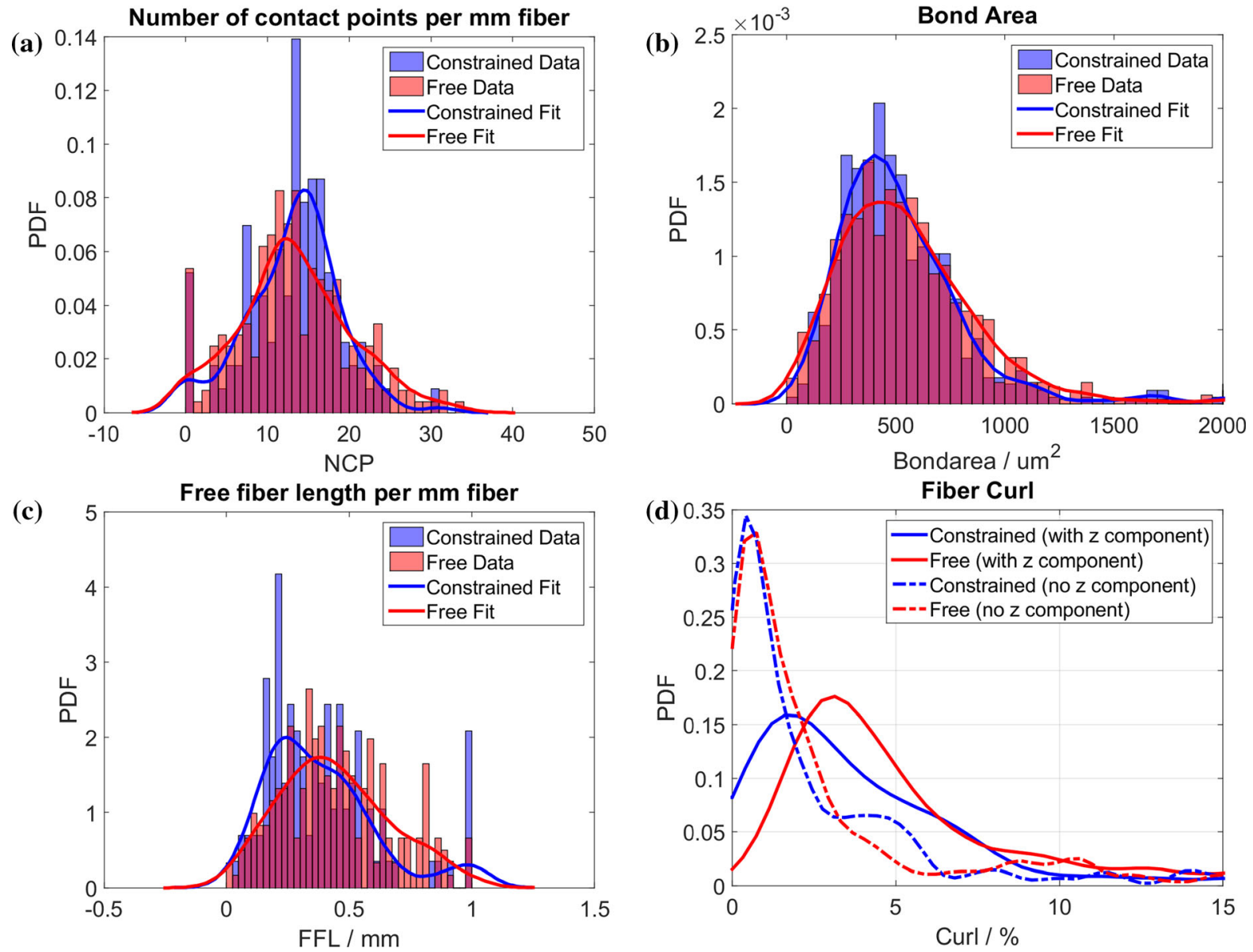

Fig. 12 This figure shows the relevant network parameters extracted from $\mu$-tomography data. The distributions of a and c were normalized by the density of the investigated area and adjusted to resemble a density of $300 \mathrm{~kg} / \mathrm{m}^{3}$. The statistics can

Comparing the freely dried (red) to the restrained dried (blue) sheet one can see that the fibers in the freely dried network exhibit a somewhat higher curvature than the constrained data for both, the fiber segments between the bonds and the fiber segments over the bonds.

\section{Conclusions}

In this work we compared free- and restrained dried labsheets with respect to the development of the mechanical properties during drying on the macroscale and changes in the fiber structure on the microscale.

be found in Table 4. No statistical difference was found for NCP (a), bond area (b) and in-plane (without z-component) fiber curl (d). Statistically significant differences were found for FFL (c) and out-of-plane (with z-component) fiber curl (d)

On the macroscale we provide an extensive study on tensile stiffness and -strength as well as the hygroexpansion coefficient during the drying process, and the effects of restrained/free drying. The (elastic) drying stresses developed during drying were found to be far below the yield limit at a given moisture, which has profound consequences. First this means that the fiber shrinkage seen in free drying occurs due to creep rather than plasticity. Second the total deformation during free drying of paper mainly consists of inelastic, creep deformation. The exact partition of creep strains between inter-fiber sliding and fiber deformation cannot be revealed with the methods used in this study, however it can be assumed that both are affected as found by Nanko and Wu (1995). 


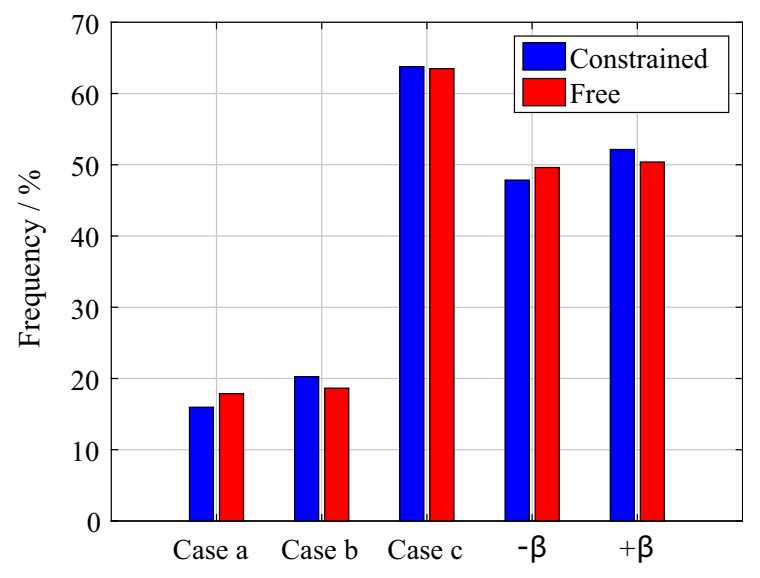

Fig. 13 Analysis of the fiber bending around bond sites in the network. The labeling refers to the explanation in Fig. 3. The data of Case a (bending towards bond), Case b (bending away from bond) and Case c (mixed) sums up to $100 \%$ as does the data for the interface angle $\beta$. Independent from drying cases $\mathrm{a}$ and $\mathrm{b}$ are much less likely to occur than the Case c. The statistics for the interface angle $\beta$ shows that in total it is equally likely that a fiber end bends towards the bond $+\beta$ like away from the bond $-\beta$

The $\mu$-CT based analysis of the paper microstructure structure revealed that fiber bond area, number of fiber-fiber contact points and in-plane fiber curl are equivalent for free- and constrained dried sheets. When including z-directional curl however, a significant increase in fiber curl was observed for the freely dried sheets. Despite the higher out-of-plane curl, our observations suggest that the changes in the interface angle between the fibers are not significantly affected by the drying strategy. This observation does not support the hypothesis presented by Uesaka (1994) in which the increased hygroexpansivity of the freely dried sheets was attributed to the changes in the bond region. As previously noted, an alternative explanation of the higher hygroexpansion of the freely dried sheets based on the interaction between stresses and orientation of both the cellulose and the hemicelluloses chains is offered by Salmén et al. (1987).

Despite being at nearly percolation density, the considered unpressed sheets still showed considerable hygroexpansion, meaning that even with a sparse network structure, the prevention of the hygroexpansion in response to changing humidity can only be done with barrier solutions.

Another relevant finding was that fiber curvature around bonding sites is different from what is commonly conveyed, Fig. 14. Usually a configuration like Fig. 3a is suggested, where fibers around the bonding region are curved towards the bond. We found that it is equally likely that the fiber is curved towards the bond (case a) like that it is curved away from the bond (case b). Surprisingly the most frequent case is c, a mixed configuration with one end of the fiber curved towards-, and one end of the fiber curved away from the bond. The overall configuration of bonds is the same in free and restrained dried sheets. We believe that this more realistic view of the bond configurations in sheets could change the micromechanical mechanisms suggested for stress transfer in fiber bonds during hygroexpansion and -shrinkage of paper.

The employed image analysis with the resolution of $0.7 \mu \mathrm{m}$ was unable to detect distinguishable differences in the bonded area of sheets subjected to different drying strategies. Although this is against speculations the bonded area being larger for freely dried sheets, Wahlström et al. (2000), the resolution of the test might be insufficient for detecting the true differences.

The observed decrease in overall fiber curl as well as lower fiber segment angles and bond interface

Table 5 Comparison of the interface angles split up in the different cases as described in Figs. 13 and 14

\begin{tabular}{|c|c|c|c|c|c|c|}
\hline Interface angle (free) & Case a & Case b & Case $\mathrm{c}$ (negative) & Case $\mathrm{c}$ (positive) & Negative side & Positive side \\
\hline Mean & 7.0 & -8.1 & -4.9 & 4.8 & -5.6 & 5.3 \\
\hline Standard deviation & \pm 3.8 & \pm 5.5 & \pm 4.0 & \pm 3.9 & \pm 4.5 & \pm 4.0 \\
\hline Error (95\% Conf.Int.) & \pm 0.46 & \pm 0.67 & \pm 0.26 & \pm 0.26 & \pm 0.26 & \pm 0.23 \\
\hline \multicolumn{7}{|c|}{ Interface angle (constrained) } \\
\hline Mean & 5.9 & -6.3 & -3.9 & 3.8 & -4.4 & 4.3 \\
\hline Standard deviation & \pm 3.3 & \pm 4.5 & \pm 3.2 & \pm 3.1 & \pm 3.7 & \pm 3.3 \\
\hline Error (95\% Conf.Int.) & 0.48 & 0.73 & 0.26 & 0.26 & \pm 0.27 & \pm 0.23 \\
\hline
\end{tabular}



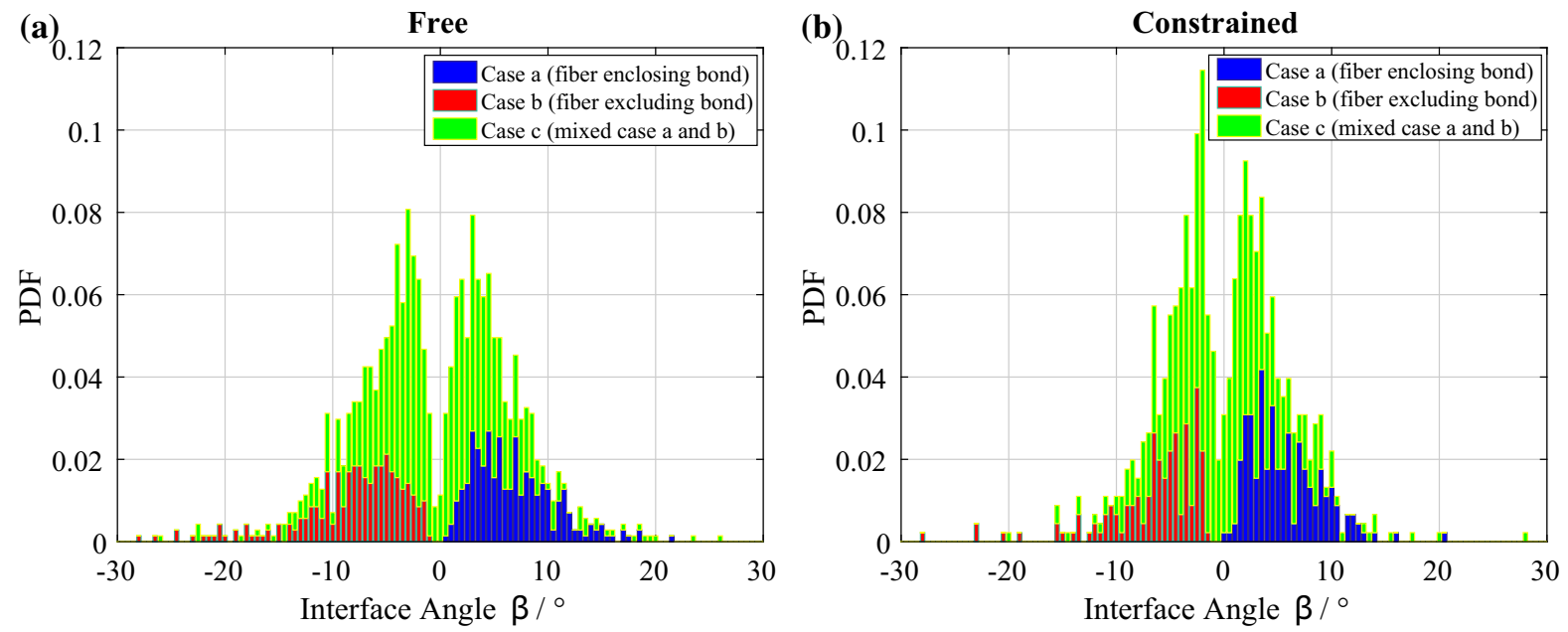

Fig. 14 Interface Angle $\beta$ distribution displayed with the information of the cases seen in Fig. 13

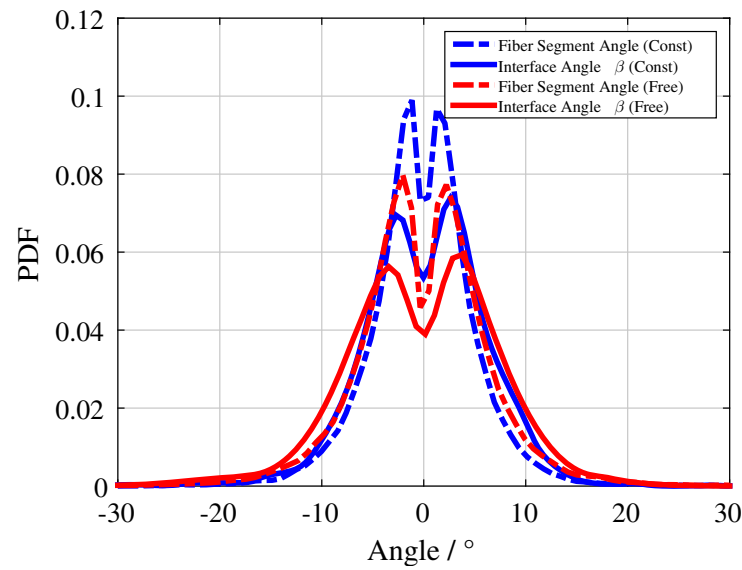

Fig. 15 Comparison of the interface angle $(\beta)$ exhibited by the fibers at the bond sites to the natural curvature of the fibers Fiber Segment Angle at the length scale of an average bond

angles $\beta$ for restrained dried sheets goes well in line with the concept of fiber activation during drying (Wahlström et al. 2000; Mäkelä 2009). The straightened fibers in case of the restrained drying can be interpreted as the manifestation of the network activation, and creating the observed increase in Emodulus and tensile strength. Finally we have been able to demonstrate a small, yet measurable fiber curvature increase in terms of interface angle $\beta$ around fiber bonding regions (Fig. 15), which has been speculated to exist for a long time (Page and Tydeman 1962; Uesaka 1990; Nanko and Tada 1995).
Acknowledgments The authors thank the Austrian Federal Ministry of Economy, Family and Youth and the Austrian National Foundation for Research, Technology and Development for their financial support. Also the industrial partners Mondi, Canon Production Printing, SIG Combibloc and Kelheim Fibres are acknowledged. The financial support of Vinnova (Grant Number 2019-02591) is acknowledged. Finally the authors thank Pascal Pucher and Wolfgang Fischer for valuable discussions and help with some of the experiments.

Open Access This article is licensed under a Creative Commons Attribution 4.0 International License, which permits use, sharing, adaptation, distribution and reproduction in any medium or format, as long as you give appropriate credit to the original author(s) and the source, provide a link to the Creative Commons licence, and indicate if changes were made. The images or other third party material in this article are included in the article's Creative Commons licence, unless indicated otherwise in a credit line to the material. If material is not included in the article's Creative Commons licence and your intended use is not permitted by statutory regulation or exceeds the permitted use, you will need to obtain permission directly from the copyright holder. To view a copy of this licence, visit http://creativecommons.org/licenses/by/4.0/.

Funding Open access funding provided by Graz University of Technology.

\section{References}

Borodulina S, Kulachenko A, Wernersson EL, Hendriks CLL (2016) Extracting fiber and network connectivity data using microtomography images of paper. Nordic Pulp Pap Res J 31(3):469-478 
Bosco E, Bastawrous MV, Peerlings RH, Hoefnagels JP, Geers MG (2015) Bridging network properties to the effective hygro-expansivity of paper: experiments and modelling. Philos Mag 95(28-30):3385-3401

Gozutok Z, Kinj O, Torun I, Ozdemir AT, Onses MS (2019) One-step deposition of hydrophobic coatings on paper for printed-electronics applications. Cellulose 26(5):3503-3512

Haslach HW Jr (1996) A model for drying-induced microcompressions in paper: buckling in the interfiber bonds. Compos B Eng 27(1):25-33

Jentzen CA (1964) The effect of stress applied during drying on some of the properties of individual pulp fibers. Ph.D. thesis, Georgia Institute of Technology

Joffre T, Isaksson P, Dumont PJ, Du Roscoat SR, Sticko S, Orgéas L, Gamstedt EK (2016) A method to measure moisture induced swelling properties of a single wood cell. Exp Mech 56(5):723-733

Kouko J, Retulainen E, Kekko P (2014) Influence of straining during wet pressing and drying on strength properties of paper. Nordic Pulp Pap Res J 29(3):453-461

Kulachenko A (2011) Moisture-induced deformations. In: Niskanen K (ed) Mechanics of paper products, Chap 9. Walter de Gruyter Incorporated, Berlin, pp 163-181

Larsson PA, Wågberg L (2008) Influence of fibre-fibre joint properties on the dimensional stability of paper. Cellulose 15(4):515-525

Lavrykov SA, Ramarao B, Lyne OL (2004) The planar transient hygroexpansion of copy paper: experiments and analysis. Nordic Pulp Pap Res J 19(2):183-190

Lindner M (2018) Factors affecting the hygroexpansion of paper. J Mater Sci 53(1):1-26

Mäkelä P (2009) Effect of drying conditions on the tensile properties of paper. In: Transactions of the 14th fundamental research symposium Oxford. pp 1079-1094

Mäkelä P, Nordhagen H, Gregersen ØW (2009) Validation of isotropic deformation theory of plasticity for fracture mechanics analysis of paper materials. Nordic Pulp Pap Res J 24(4):388-394

Motamedian HR, Kulachenko A (2019) Simulating the hygroexpansion of paper using a $3 \mathrm{~d}$ beam network model and concurrent multiscale approach. Int J Solids Struct $161: 23-41$

Nanko H, Tada Y (1995) Mechanism of hygroexpansion of paper. In: International paper physics conference, Niagaraon-the-Lake, Canada. pp 159-171

Nanko H, Wu J (1995) Mechanisms of paper shrinkage during drying. In: International paper physics conference, Niagara-on-the-Lake, Canada. pp 103-113

Niskanen KJ, Kuskowski SJ, Bronkhotst CA (1997) Dynamic hygroexpansion of paperboards. Nordic Pulp Pap Res J 12(2):103-110
Page DH, Tydeman P (1962) A new theory of the shrinkage, structure and properties of paper. In: Bolam F (ed) The formation and structure of paper, Technical Section British Paper and Boards Makers' Association, London vol 1. pp 397-425

Pulkkinen I, Fiskari J, Alopaeus V (2009) The effect of hardwood fiber morphology on the hygroexpansivity of paper. BioResources 4(1):126-141

Salmén L, Fellers C, Htun M (1987) The development and release of dried-in stresses in paper. Nord Pulp Pap Res J 2:44-48

Salminen L, Alava M, Heyden S, Gustafsson P-J, Niskanen K (2002) Simulation of network shrinkage. Nordic Pulp Pap Res J 17(2):105-110

Sellén C, Isaksson P (2014) A mechanical model for dimensional instability in moisture-sensitive fiber networks. J Compos Mater 48(3):277-289

Sung YJ, Keller D et al (2008) Local paper structural properties by noncontacting laser profilometry. Appita J J Tech Assoc Aust N Z Pulp Pap Ind 61(1):28

Tydeman P, Wembridge D, Page D (1966) Transverse shrinkage of individual fibres by micro-radiography. In: Consolidation of the paper web. pp 119-144

Uesaka T (1990) Hygroexpansion coefficients of paper. In: Perkins RW (ed) Mechanics of wood and paper materials. ASME, New York, pp 29-35

Uesaka T (1994) General formula for hygroexpansion of paper. J Mater Sci 29(9):2373-2377

Uesaka T, Moss C (1997) Effects of fiber morphology on hygroexpansivity of paper-a micromechanics approach. Fund Papermak Mater 1:663-679

Uesaka T, Qi D (1994) Hygroexpansivity of paper: effects of fibre-to-fibre bonding. J Pulp Pap Sci 20(6):J175-J179

Wahlström T (2009) Development of paper properties during drying. In: Ek M, Gellerstedt G, Henriksson G (eds) Pulp and paper chemistry and technology, vol 4 . Walter de Grutyer GmbH \& Co. KG, Berlin, pp 69-108

Wahlström T, Lundh A, Hansson T, Fellers C (2000) Biaxial straining of handsheets during drying-effect on delamination resistance. Nordic Pulp Pap Res J 15(3):237-242

Wernersson ELG, Borgefors G, Borodulina S, Kulachenko A (2014) Characterisations of fibre networks in paper using micro computed tomography images. Nordic Pulp Pap Res J 29(3):468-475

Publisher's Note Springer Nature remains neutral with regard to jurisdictional claims in published maps and institutional affiliations. 